\title{
A continual model of a damaged medium used for analyzing fatigue life of polycrystalline structural alloys under thermal-mechanical loading
}

Received: 7 December 2018 / Accepted: 25 May 2019 / Published online: 5 June 2019

(C) The Author(s) 2019

\begin{abstract}
The main physical laws of thermal-plastic deformation and fatigue damage accumulation processes in polycrystalline structural alloys under various regimes of cyclic thermal-mechanical loading are considered. Within the framework of mechanics of damaged media, a mathematical model is developed that describes thermal-plastic deformation and fatigue damage accumulation processes under low-cycle loading. The model consists of three interrelated parts: relations defining plastic behavior of the material, accounting for its dependence on the failure process; evolutionary equations describing damage accumulation kinetics; a strength criterion of the damaged material. The plasticity model based on the notion of yield surface and the principle of orthogonality of the plastic strain vector to the yield surface is used as defining relations. This version of defining equations of plasticity describes the main effects of the deformation process under monotone cyclic, proportional and nonproportional loading regimes. The version of kinetic equations of damage accumulation is based on introducing a scalar parameter of damage degree and energy principles, and account for the main effects of nucleation, growth and merging of microdefects under arbitrary regimes of low-cycle loading. The strength criterion of the damaged material is based on reaching a critical value of the damage degree. The results of numerically modeling cyclic thermal-plastic deformation and fatigue damage accumulation in heat-resistant alloys (Nimonic 80A, Haynes 188) under combined thermal-mechanical loading are presented. Special atten-
\end{abstract}

Communicated by Andreas Öchsner.

I. A. Volkov · L. A. Igumnov · F. dell'Isola · S. Yu. Litvinchuk · V. A. Eremeyev ( $\varangle)$

Research Institute for Mechanics, National Research Lobachevsky State University of Nizhny Novgorod, 23 Prospekt Gagarina (Gagarin Avenue) BLDG 6, Nizhny Novgorod, Russian Federation 603950

E-mail: eremeyev.victor@gmail.com

I. A. Volkov

E-mail: pmptmvgavt@yandex.ru

L. A. Igumnov

E-mail: igumnov@mech.unn.ru

F. dell'Isola

E-mail: fdellisola@gmail.com

S. Yu. Litvinchuk

E-mail: litvinchuk@mech.unn.ru

F. dell'Isola

Dipartimento Ingegneria Strutturale e Geotecnica, Università di Roma "La Sapienza”, 00184 Rome, Italy

V. A. Eremeyev

Faculty of Civil and Environmental Engineering, Gdansk University of Technology, Narutowicza 11/12, Gdańsk Wrzeszcz, 80-233 Gdańsk, Poland 
tion is paid to the issues of modeling the processes of cyclic thermal-plastic deformation and fatigue damage accumulation for complex deformation processes accompanied by the rotation of the main stress and strain tensor areas. It is shown that the present damaged medium model accurately enough for engineering purposes describes the processes of cyclic isothermal and nonisothermal deformation and fatigue damage accumulation under combined thermal-mechanical loading and makes it possible to evaluate low-cycle fatigue life of heat-resistant alloys under arbitrary deformation trajectories.

Keywords Modeling - Thermal cyclic strength - Complex deformation - Damaged medium mechanics · Fatigue life $\cdot$ Strength $\cdot$ Failure

\section{Introduction}

The general trend in the development of structures and machines of modern mechanical engineering is characterized by the increasing number of their working parameters, the decreasing metal consumption due to optimal design and use of novel high-strength materials, the increasing relative share of nonstationary loading regimes. The requirements to reliability and accident-free life of both the entire structures and their separate elements are becoming tougher. As a result, one of the main tasks of the development and exploitation of structures and machines of modern technologies at present is the task of reliable evaluation of their service life. This task is especially vital for objects with service lives of several tens of years (atomic power plants, aviation gas-turbine engines, spaceship engines, etc.). as a rule, the exploitation conditions of such structures and machines are characterized by multiparametric nonstationary thermal-mechanical loading, effects of external fields leading to the degradation of the initial strength properties of structural materials and, finally, to exhausting the life of the structural units of the object [1-7].

Effects of deformation under thermal-mechanical loading are determined by the dependence of the physical-mechanical properties of structural materials on temperature, by the presence of limitations of thermal strains and the effect of the ratio of mechanical and thermal strain rates. As frequencies and phases of variation cycles of temperature and mechanical strain tensor components as a rule do not coincide, nonisothermal cyclic loading is generally irregular, nonproportional, multiaxial and is accompanied by the rotation of the main areas of stress and strain tensors.

Cyclic nonisothermal deformation of materials, in contrast to isothermal one, is a nonbalanced cyclic process. The degree of imbalance is a function of the range of the temperature cycle and phase shift in the variation of temperature and deformation. Even in the elastic region of the tension-compression cycle, due to the dependence of the elasticity modulus on temperature (a nonlinear decreasing function of the growing temperature), the deformation process depends on the phase of the variation of the temperature and mechanical strain and is hard at the end of the cold temperature cycle and more elastic at its high-temperature end [1]. In the case of nonisothermal elastoplastic deformation, the deformation process will have a more complex character as the elastoplastic behavior of the material depends on temperature.

As a rule, a volumetric thermal strain results in a multiaxial deformation process. This leads to new sources of imbalance: the stressed-strained state in any point of the cycle differs from that in other points. Phasing by varying the temperature and mechanical strain is one of the main factors also connected with thermal fatigue.

The multiaxial nature of the stressed-strained state also causes imbalances in thermal-mechanical loading. A combined effect of mechanical and thermal loads results, as a rule, in substantial rotation of the main areas of stress and strain tensors (nonproportional loading), which, in the presence of plastic deformation leads to noncoaxiality of stress and total and plastic strains tensors. To model such processes, reliable models of cyclic thermal plasticity are required.

Nonisothermal deformation of structural materials, as compared with isothermal one, is characterized by a number of specific features [1]:

- the nonlinear stress-strain relation even in the elastic region, as elasticity moduli depend on temperature;

- the imbalanced nature of the diagrams for symmetric cycles of variation of mechanical strain and strong dependence of the form of the stress-strain hysteresis loop on the combination of the variation phases of temperature and mechanical strain.

Thermal-mechanical fatigue cycling may initiate damage mechanisms that do not develop under the effect of isothermal fatigue. The main parameters determining the rates of thermal-mechanical fatigue processes are: temperature gradients, maximal temperature of the cycle, geometric characteristics and conditions of 
joining of structural elements, physical-mechanical characteristics of materials (especially the linear expansion coefficient), inhomogeneity (anisotropy) of the structural composition of such materials.

Thus, despite the apparent similarity of the phenomena of isothermal and nonisothermal types of fatigue, the results on isothermal low-cycle fatigue have to be used with due care when evaluating nonisothermal fatigue life.

Damage and failure of materials is, for the most part, caused by nucleation of microdefects, their growth and merging to form macroscopic cracks. The tasks of describing the mechanical behavior of such microdefects are as important as the tasks of describing the growth of macrodefects (cracks); so, in the recent years, a new scientific direction, mechanics of damaged media (MDM), is being successfully developed to solve these tasks [8-16].

In [12-16], in the framework of MDM, a mathematical model was developed that describes processes of cyclic thermal-plastic deformation and fatigue damage accumulation in structural materials (metals and their alloys) under multiaxial nonproportional paths of combined thermal-mechanical loading. To assess qualitatively and quantitatively the reliability of the model, the present paper investigates the effect of the laws of change of mechanical strength and temperature (the type of deformation trajectory) on fatigue life of heat-resistant alloys (Nimonic 80A, Haynes 188) under nonproportional regimes of thermal-mechanical loading.

The methods presented in this paper can also be suitably applied to a large class of metamaterials (see $[17,18]$ for a general discussion about metamaterials). In particular, continuum models for describing the emerging of damage and fracture have been investigated in [19-22].

\section{The constitutive relations of mechanics of damaged media}

The damaged medium model developed in [12-16] consists of three interrelated parts:

- relations determining thermal-plastic behavior of materials, accounting for its dependence on the failure process;

- evolutionary equations describing damage accumulation kinetics;

- a strength criterion of the damaged material.

(a) Constituting relations of plasticity

The governing relations of plasticity are based on the following main assumptions [23]:

- the tensor components of strains $e_{i j}$ and strain rates $\dot{e}_{i j}$ include elastic $e_{i j}^{e}, \dot{e}_{i j}^{e}$ and plastic strains $-e_{i j}^{p}, \dot{e}_{i j}^{p}$, i. e., reversible and irreversible components;

- the initial yield surface for different temperatures is described by a von Mises-type surface. The evolution of the yield surface is described by the variation of its radius $C_{p}$ and the displacement of its center $\rho_{i j}$;

- the body changes its volume elastically;

- initially isotropic media are considered. Only the anisotropy caused by processes of plasticity are accounted for;

- processes characterized by small deformations are considered.

In the elastic region, the correlation between spherical and deviatoric components of stress and strain tensors and of their rates is established with the Hooke law:

$$
\sigma=3 K\left[e-\alpha\left(T-T_{0}\right)\right], \quad \sigma_{i j}=2 G e_{i j}^{\prime e}, \quad \dot{\sigma}=3 K(\dot{e}-\dot{\alpha} T-\alpha \dot{T})+\frac{\dot{K}}{K} \sigma, \quad \dot{\sigma}_{i j}^{\prime}=2 G \dot{e}_{i j}^{\prime e}+\frac{\dot{G}}{G} \sigma_{i j}^{\prime},
$$

where $T$ is temperature, $T_{0}$ is an initial (referential) temperature, $K(T)$ is the bulk modulus, $G(T)$ is the shear modulus, $\alpha(T)$ is the coefficient of linear thermal expansion of the material.

To model the effects of monotone and cyclic deformation, a yield surface is introduced:

$$
F_{s}=S_{i j} S_{i j}-C_{p}^{2}=0, \quad S_{i j}=\sigma_{i j}^{\prime}-\rho_{i j} .
$$

To describe complex cyclic deformation regimes in the stress space, a cyclic 'memory' surface is introduced. The equation of the 'memory' surface is: 


$$
F_{\rho}=\rho_{i j} \rho_{i j}-\rho_{\max }^{2}=0,
$$

where $\rho_{\max }$ is maximal value of modulus $\rho_{i j}$ during the loading history.

It is assumed that the evolutionary equation for the yield surface radius has the following structure:

$$
\begin{aligned}
\dot{C}_{p} & =\left[q_{\chi} H\left(F_{\rho}\right)+a\left(Q_{s}-C_{p}\right) \Gamma\left(F_{\rho}\right)\right] \dot{\chi}+q_{3} \dot{T} \\
C_{p} & =C_{p}^{0}+\int_{0}^{t} \dot{C}_{p} d t, \quad \dot{\chi}=\left(\frac{2}{3} \dot{e}_{i j}^{p} \dot{e}_{i j}^{p}\right)^{1 / 2}, \quad \chi_{m}=\int_{0}^{t} \dot{\chi} H\left(F_{\rho}\right) d t, \quad \chi=\int_{0}^{t} \dot{\chi} d t . \\
q_{\chi} & =\frac{q_{2} A \psi_{1}+(1-A) q_{1}}{A \psi_{1}+(1-A)}, \quad Q_{s}=\frac{Q_{2} A \psi_{2}+(1-A) Q_{1}}{A \psi_{2}+(1-A)}, \quad 0 \leq \psi_{i} \leq 1, \quad i=1,2 . \\
A & =1-\cos ^{2} \theta, \quad \cos \theta=n_{i j}^{e} n_{i j}^{s}, \quad n_{i j}^{e}=\frac{\dot{e}_{i j}^{\prime}}{\left(\dot{e}_{i j}^{\prime} \dot{e}_{i j}^{\prime}\right)^{1 / 2}}, \quad n_{i j}^{s}=\frac{S_{i j}}{\left(S_{i j} S_{i j}\right)^{1 / 2}}, \\
H\left(F_{\rho}\right) & =\left\{\begin{array}{l}
1, F_{\rho}=0 \wedge \rho_{i j} \dot{\rho}_{i j}>0 \\
0, F_{\rho}<0 \vee \rho_{i j} \rho_{i j} \leq 0
\end{array}\right\}, \quad \Gamma\left(F_{\rho}\right)=1-H\left(F_{\rho}\right),
\end{aligned}
$$

where $q_{1}, q_{2}, q_{3}$ are experimentally determined constitutive isotropic hardening moduli, $Q_{1}$ and $Q_{2}$ are cyclic isotropic hardening moduli, $a$ is a constant determining the rate of the stationing process of the hysteresis loop of cyclic deformation of the material, $Q_{s}$ is stationary value of the yield surface radius for the assigned $\rho_{\max }$ and $T, C_{p}^{0}$ is initial value of the yield surface radius $[12,23]$.

The evolution of internal variable $\rho_{i j}$ is assumed to have the form [24]:

$$
\begin{aligned}
& \dot{\rho}_{i j}=\tilde{g}_{1} \dot{e}_{i j}^{p}-g_{2} \rho_{i j} \dot{\chi}+\dot{\rho}_{i j}^{*}+g_{5} \rho_{i j}\langle\dot{T}\rangle, \quad \rho_{i j}=\int_{0}^{t} \dot{\rho}_{i j} d t, \\
& \tilde{g}_{1}=g_{1}+k_{1}\left(1-e^{-k_{2} \chi_{m}}\right)\langle\cos \beta\rangle, \quad\langle\cos \beta\rangle=\frac{\dot{\rho}_{i j} \rho_{i j}}{\left(\dot{\rho}_{i j} \dot{\rho}_{i j}\right)^{1 / 2}\left(\rho_{i j} \rho_{i j}\right)^{1 / 2}}, \\
& \dot{\rho}_{i j}^{*}=g_{3} \dot{e}_{i j}^{p} H\left(F_{\rho}\right)-g_{4} \rho_{i j} \dot{\chi},
\end{aligned}
$$

where $g_{1}, g_{2}, g_{3}, g_{4}, g_{5}, k_{1}$ and $k_{2}$ are experimentally determined material parameters.

For nonsymmetric hard and soft types of cyclic loading, Eq. (7) describes the processes of placing and ratcheting of the cyclic plastic hysteresis loop with the help of term $\dot{\rho}_{i j}^{*}$. If $g_{3}=g_{4}=k_{1}=0$ in (7), one has a special case of Eq. (7) that is the Armstrong-Frederik-Kadashevich equation [23]:

$$
\dot{\rho}_{i j}=g_{1} \dot{e}_{i j}^{p}-g_{2} \rho_{i j} \dot{\chi} .
$$

To describe the evolution of the 'memory' surface, it is necessary to formulate an equation for $\rho_{\max }$ :

$$
\dot{\rho}_{\max }=\frac{\left(\rho_{i j} \dot{\rho}_{i j}\right) H\left(F_{\rho}\right)}{\left(\rho_{m n} \rho_{m n}\right)^{1 / 2}}-g_{2} \rho_{\max } \dot{\chi}-g_{5} \rho_{\max } \dot{T} .
$$

The plastic strain rate components obey the rule of orthogonality of the plastic strain rate vector to the yield surface in the loading point:

$$
\dot{e}_{i j}^{p}=\lambda S_{i j}
$$

where $\lambda$ is proportionality coefficient determined from the condition that a new yield surface passes through the end of the stress deviator vector at the end of the loading stage.

At the stage of defects scattered over the volume, the effect of the damage degree on the physical-mechanical properties of the material is observed. This effect can be taken into account by introducing effective stresses:

$$
\tilde{\sigma}_{i j}^{\prime}=F_{1}(\omega) \sigma_{i j}^{\prime}=\frac{G}{\tilde{G}} \sigma_{i j}^{\prime}=\frac{\sigma_{i j}^{\prime}}{(1-\omega)\left[1-\frac{(6 K+12 G)}{(9 K+8 G)} \omega\right]},
$$




$$
\tilde{\sigma}=F_{2}(\omega) \sigma=\frac{\kappa}{\tilde{K}} \sigma=\frac{\sigma}{4 G(1-\omega) /(4 G+3 K \omega)},
$$

where $\tilde{G}, \tilde{K}$ are effective moduli of elasticity defined by McKenzie formulas [13].

Effective variable $\tilde{\rho}_{i j}$ is determined in a similar way:

$$
\tilde{\rho}_{i j}=F_{1}(\omega) \rho_{i j}
$$

\section{(b) Evolutionary equation of fatigue damage accumulation}

In the framework of the mechanics of damaged media, the final failure stage corresponds to the nucleation of a macroscopic crack $(\sim 0.1 \mathrm{~cm})$, that is, leading to material discontinuity, which is rather large at the level of heterogeneity of the material (grain, structure blocks, etc.).

The simplest visual physical measure of material damage is the relative volumetric fraction of defects in the reference elementary volume of the material $\omega$ [13]: $\omega=V / V_{f}, 0 \leq \omega \leq 1$, where $V$ is the current volumetric fraction of defects, $V_{f}$ is the critical volumetric fraction, corresponding to nucleation of a macrocrack in this volume of the material (complete loss of the bearing capacity of the material in this elemental volume).

To formulate evolutionary equations for $\omega$, we use an approach based on establishing the correlation of velocities $\dot{\omega}$ with certain mechanical parameters, depending on the material deformation process, the critical value of which determines the moment of complete failure of the elementary volume of the material. The most effective approach to solving this problem is the energy approach $[3,8,9,12,13]$.

It is not a trivial task to extract from the total dissipation energy in the volume the part of it that is directly spent on the damage formation and accumulation.

It is established (see $[12,13]$ and the references therein) that, at low-cycle fatigue, the energy, spent on nucleation of defect during transient elastoplastic deformation, correlates well with the work of $W$ the microstress tensor (center coordinates of the yield surface $F$ ) $\rho_{i j}$ on plastic deformations $e_{i j}^{p}$ :

$$
\dot{W}=\rho_{i j} \dot{e}_{i j}^{p}, \quad W=\int \rho_{i j} d e_{i j}^{p} .
$$

The multiaxial nature of stress state significantly affects the durability of the material, and this effect manifests in two ways: the effect of multiaxial nature under proportional loading (when all components of the strain tensor vary proportionally to one parameter) and the rotation effect of the main stress tensor areas (when stress components vary out of phase).

Experimental and theoretical studies of the influence of multiaxial nature in other types of stress states (biaxial tension-compression, triaxial tension) showed a significant effect of volumetric stressed state of the material on its durability, characterized by the intensity of the stress tensor $\sigma_{u}$ and its ball (hydrostatic) component $\sigma[8,9,12,13]$.

Parameter $\beta=\sigma / \sigma_{u}$ or functions of $\beta$ are used as parameters of the volumetric stressed state. Summarizing the data available in the literature, it can be confirmed that the rate of damage accumulation $\dot{\omega}$ depends on volumetric stressed state, characterized by some function $f_{1}(\beta)$ : $\dot{\omega}$ increases with $\beta>0$ and $\beta \rightarrow+\infty$ (threedimensional tension) and decreases with $\beta<0$ and $\beta \rightarrow-\infty$ (in this case partial healing of the accumulated damage may occur). With $\beta=0$ (pure shift, $\sigma=0$ ), the normalized function $f_{1}(\beta)$ should be equal to 1 .

The effect of nonproportional loading, under which the components of the stress-strain tensors change out of phase (the main tensor areas rotate), and the stress and plastic strain tensors are not coaxial, is as follows $[9,11,12]$ :

- the shape of the deformation path is a parameter that significantly affects the fatigue life;

- structural materials exhibit complex cyclical behavior under multiaxial nonproportional loading conditions —additional cyclic hardening or softening;

- under nonproportional cyclic loading, the criterion of equivalent deformations or strain intensity $e_{u}$ are not equivalence criteria and may lead to nonconservative evaluations.

Summarizing the above results, the general structure of an evolutionary equation of the damage accumulation in elementary volume of the material can have the following form [12-16]:

$$
\dot{\omega}=f_{1}(\beta) f_{2}(\omega) f_{3}(W) f_{4}(\dot{W}) .
$$


where functions $f_{i}, i=1 \ldots 4$ account for: the volumetric stressed state $\left(f_{1}(\beta)\right)$, level of accumulated damage $\left(f_{2}(\omega)\right)$, accumulated relative damage energy, spent on nucleation of defects $\left(f_{3}(W)\right)$ and rate of change of damage energy $\left(f_{4}(\dot{W})\right)$.

In (16):

$$
\begin{aligned}
& f_{1}(\beta)=\exp (\beta), \quad f_{2}(\omega)=\left\{\begin{array}{l}
0, \quad W_{\leq} W_{a}, \\
\omega^{1 / 3}(1-\omega)^{2 / 3} \wedge W_{>} W_{a} \wedge \omega \leq 1 / 3, \\
\frac{\sqrt[3]{16}}{9} \omega^{-1 / 3}(1-\omega)^{-2 / 3} \wedge W_{>} W_{a} \wedge \omega>1 / 3,
\end{array}\right. \\
& f_{3}(W)=\frac{W_{-} W_{a}}{W_{f}-W_{a}}, \quad f_{4}(\dot{W})=\dot{W} /\left(W_{f}-W_{a}\right) .
\end{aligned}
$$

where $\beta$ is parameter of volumetric stressed state, $W_{a}$ is value of the damage energy at the end of the stage of nucleation of scattered defects for LCF, and $W_{f}$ is value of the energy corresponding to the nucleation of a macroscopic crack.

The duration of the microdefect nucleation phase will be related with the value of parameter $W_{a}$.

When the size of microdefects becomes comparable with the average distance between them, the merging process (breakage of the remaining continuous spaces between the defects) begins. The present paper was not aimed at constructing a detailed model of defect merging; instead, this process was accounted for by formulating the kinetic equation in such a way (due to using term $f_{2}(\omega)$ ) that when the damage degree reaches the value of $\omega=1 / 3$ relation $\dot{\omega}=f_{1}(\omega)$ accounts for the avalanche-like increase in the damage degree value.

\section{c) The strength criterion of the damaged material}

The condition when damage degree $\omega$ reaches its critical value

$$
\omega=\omega_{f} \leq 1
$$

is taken as the criterion of termination of the phase of growth of scattered microdefects.

\section{Numerical results}

The results on thermal cyclic deformation of the heat-resistant nickel alloy Nimonic 80A are presented in [25]. Isothermal (Fig. 1a) and nonisothermal (Fig. 1b, c) tests were conducted with hard loading, the amplitude of strain $e_{11}$ in the tests with constant temperature $T$ being $1 \%$. For the tests with a varying temperature, the results are given for the amplitudes from 0.45 to $1 \%$. The temperature in nonisothermal tests varied both in-phase (Fig. 1b) and in counter-phase (Fig. 1c) relative to the variation of the strains.

The main physical-mechanical characteristics of the heat-resistant nickel alloy Nimonic 80A and the material parameters of the damaged medium model $[12,13]$ are listed in Tables 1,2 and 3.

When conducting the experiments, the selected samples should ensure a uniform distribution of stress, strain and temperatures within the operating part of the sample. If this condition is met, then, for numerical modeling of experimental processes, there is no need in formulation of the initial-boundary problem with the statement of boundary conditions and the use of computer complexes of finite element analysis [12,13]. The calculation of the processes of deformation and failure of laboratory samples is carried out by numerical integration

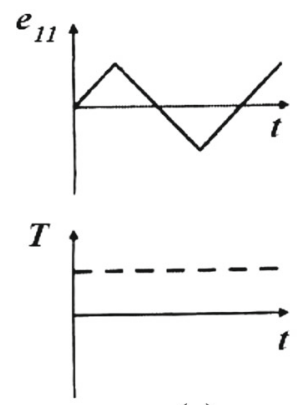

(a)
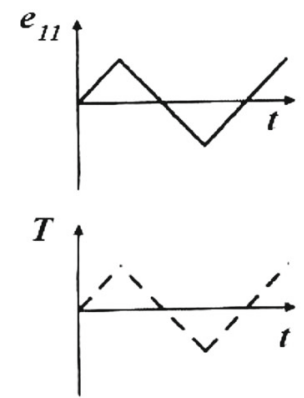

(b)
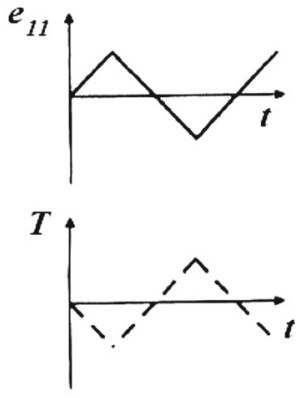

(c)

Fig. 1 Laws of change of axial strain $e_{11}$ and temperature $T$ 


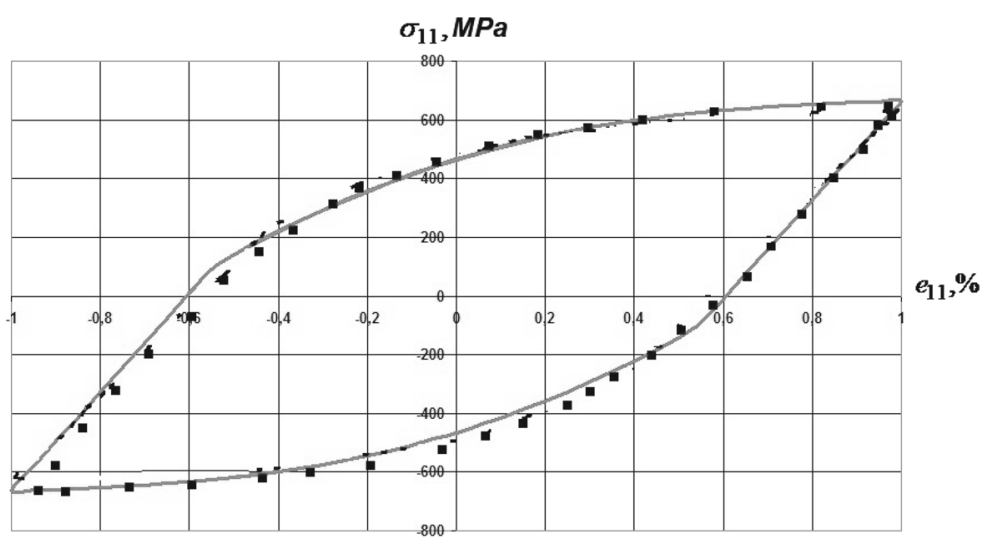

(a)

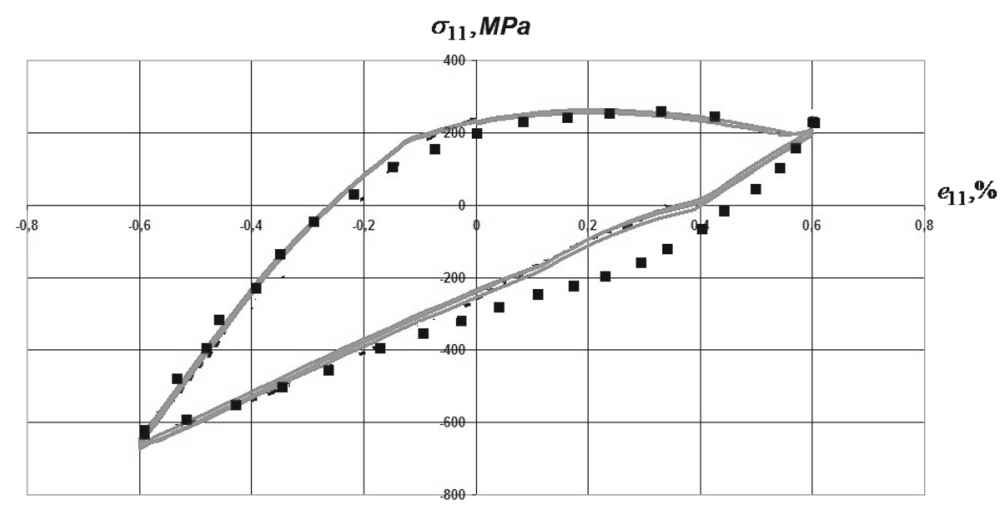

(b)

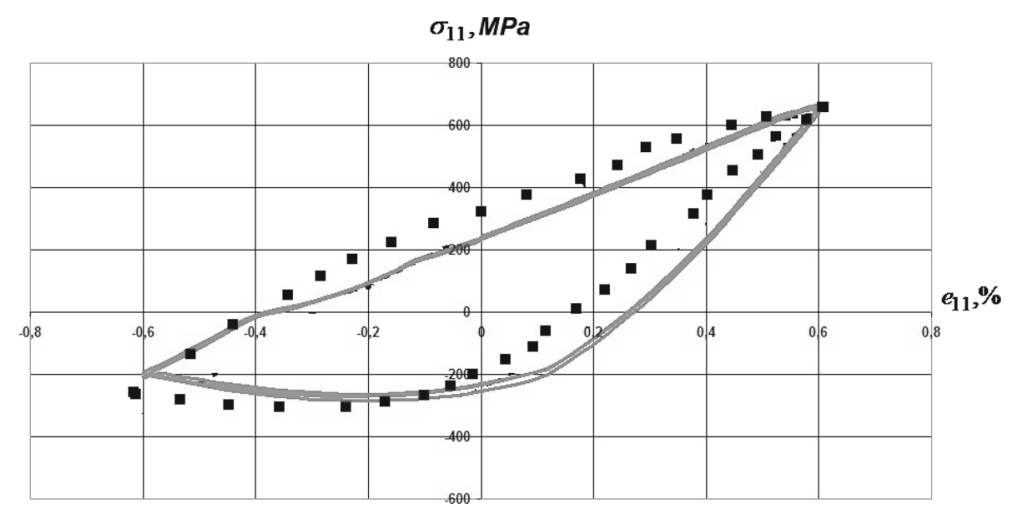

(c)

Fig. 2 Stabilized cyclic deformation loops

of the defining relations of the MDM (1)-(18). In numerical simulation of experimental data, experimental deformation trajectories (laws of changes in the components of the strain tensor $e_{i j}(t)$ and temperature $T(t)$ ) are specified, while stress trajectories $\sigma_{i j}(t)$ and damage values $\omega(t)$ are obtained by integrating the defining relations of MDM.

Figure 2 a compares the results of modeling and the experimental data for a stabilized cyclic deformation loop for the temperature of $700^{\circ} \mathrm{C}$ (the loading type is shown in Fig. 1a), for the strain amplitude of $1 \%$. Here and in what follows the markers show the experimental results, and the solid lines depict the numerical results obtained using the MDM model introduced by the authors of $[12,13]$.

Figure $2 \mathrm{~b}$ presents the comparison of the stabilized loops of cyclic nonisothermal deformation for in-phase loading (see Fig. 1b): the strain amplitude is $0.6 \%$, the maximal temperature in the cycle was $823^{\circ} \mathrm{C}$ (achieved in tension), the minimal temperature in the cycle was $571{ }^{\circ} \mathrm{C}$ (achieved in compression). 


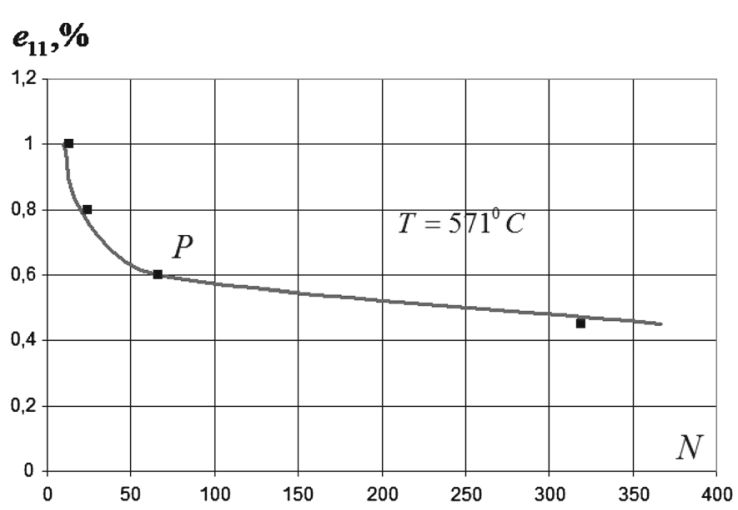

(a)

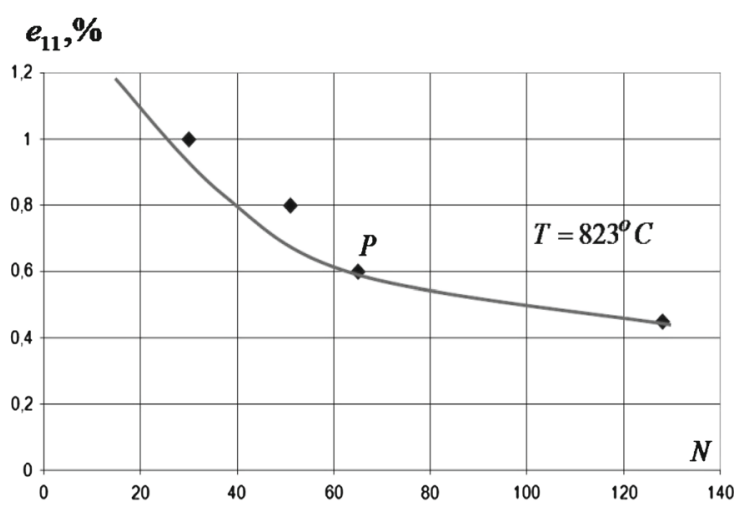

(b)

Fig. 3 Isothermal fatigue curves of the heat-resistant alloy Nimonic 80A

Table 1 The main physical-mechanical characteristics and the material parameters of the damaged medium model of the heatresistant alloy Nimonic 80A

\begin{tabular}{llll}
\hline Characteristics & Temperature $T\left({ }^{\circ} \mathrm{C}\right)$ & \\
\cline { 2 - 4 } & 571 & 700 & 823 \\
\hline$K(\mathrm{MPa})$ & 150,500 & 139,666 & 106,000 \\
$G(\mathrm{MPa})$ & 70,450 & 64,461 & 48,333 \\
$\alpha\left(1 /{ }^{\circ}\right)$ & 0.0000148 & 0.0000155 & 0.0000164 \\
$C_{p}^{\circ}(\mathrm{MPa})$ & 384 & 363 & 340 \\
$g_{1}(\mathrm{MPa})$ & 70,850 & 56,300 & 15,500 \\
$g_{2}$ & 308 & 290 & 271 \\
$a$ & 65 & 58 & 45 \\
$W_{f}\left(\mathrm{MJ} / \mathrm{m}^{3}\right)$ & 41.5 & 39 & 35.5 \\
$W_{a}\left(\mathrm{MJ} / \mathrm{m}^{3}\right)$ & 0 & 0 & 0 \\
\hline
\end{tabular}

Figure 2c compares the results of modeling the nonisothermal cyclic loading for the counter-phase loading (see Fig. 1c) (the strain amplitude and the maximal and minimal temperature values are the same as in the previous case). The 67-th and 68-th loading half-cycles are shown as stabilized cyclic deformation loops in all the three cases.

It can be seen that the model of cyclic thermal-plastic deformation presented in the paper adequately describes the experimental results and can be used in future for analyzing the kinetics of the fatigue damage accumulation process under cyclic in-phase and counter-phase thermal-mechanical effects.

To assess the applicability of the introduced evolutionary equation of fatigue damage accumulation (16) and to analyze the effect of the deformation trajectory on low-cycle fatigue, fatigue life of specimens made of the heat-resistant alloy Nimonic 80A was analyzed for different laws of change of axial deformation $e_{11}$ and temperature $T$ (see Fig. 1). The numerical results were compared with the available test data [25]. The material parameters of the evolutionary equation of damage accumulation were determined using point $P$ on the fatigue curve for isothermal cyclic deformation (Fig. 3). The material parameters of the evolutionary equation of damage accumulation (16) of the heat-resistant alloy Nimonic 80A are summarized in Table 1.

The numerical results are compared with the available test data in Figs. 3, 4 and Table 4.

Figure $3 \mathrm{a}, \mathrm{b}$ depicts the isothermal fatigue curves, and Fig. $4 \mathrm{a}, \mathrm{b}$ the nonisothermal fatigue curves for the in-phase and counter-phase loading regimes; the isothermal tests for the different temperatures $T=571^{\circ} \mathrm{C}$ and $823^{\circ} \mathrm{C}$ (Fig. 3a, b, respectively) and the nonisothermal in-phase (Fig. 4a) and counter-phase (Fig. 4b). The solid lines represent the corresponding calculated fatigue life curves. The experimental results are shown by square markers.

The numerical and experimental results show that:

- for known parameters of cyclic thermal plasticity equations, material parameters of evolutionary equations of damage accumulation (16-18) are determined using a single experimental point $P$ on the fatigue curve 
Table 2 Modulus of monotone isotropic hardening $q_{1}(\mathrm{MPa})$ as a function of the length of the plastic deformation path on monotone parts $\chi_{m}$ for the heat-resistant alloy Nimonic $80 \mathrm{~A}\left(q_{2}=0\right)$

\begin{tabular}{|c|c|c|c|c|c|c|c|c|c|}
\hline \multicolumn{10}{|c|}{$T=571^{\circ} \mathrm{C}$} \\
\hline $\begin{array}{l}\chi_{m} \\
q_{1}\end{array}$ & $\begin{array}{l}0 \\
-1981\end{array}$ & $\begin{array}{l}0.0005 \\
-5362\end{array}$ & $\begin{array}{l}0.001 \\
-8255\end{array}$ & $\begin{array}{l}0.002 \\
-11,002\end{array}$ & $\begin{array}{l}0.003 \\
-8321\end{array}$ & $\begin{array}{l}0.004 \\
-7006\end{array}$ & $\begin{array}{l}0.005 \\
-8243\end{array}$ & $\begin{array}{l}0.0075 \\
-14,420\end{array}$ & $\begin{array}{l}0.01 \\
-20,600\end{array}$ \\
\hline \multicolumn{10}{|c|}{$T=700^{\circ} \mathrm{C}$} \\
\hline $\begin{array}{l}\chi_{m} \\
q_{1} \\
\end{array}$ & $\begin{array}{l}0 \\
2666 \\
\end{array}$ & $\begin{array}{r}0.0005 \\
-3667 \\
\end{array}$ & $\begin{array}{l}0.001 \\
-4202 \\
\end{array}$ & $\begin{array}{l}0.002 \\
-3847 \\
\end{array}$ & $\begin{array}{l}0.003 \\
-3049 \\
\end{array}$ & $\begin{array}{l}0.004 \\
-8346 \\
\end{array}$ & $\begin{array}{l}0.005 \\
-8112 \\
\end{array}$ & $\begin{array}{l}0.0075 \\
3072 \\
\end{array}$ & $\begin{array}{l}0.01 \\
14,260 \\
\end{array}$ \\
\hline \multicolumn{10}{|c|}{$T=823{ }^{\circ} \mathrm{C}$} \\
\hline $\begin{array}{l}\chi_{m} \\
q_{1}\end{array}$ & $\begin{array}{l}0 \\
-4974\end{array}$ & $\begin{array}{l}0.0005 \\
-4405\end{array}$ & $\begin{array}{l}0.001 \\
-3692\end{array}$ & $\begin{array}{l}0.002 \\
-2124\end{array}$ & $\begin{array}{l}0.003 \\
-3716\end{array}$ & $\begin{array}{l}0.004 \\
-3463\end{array}$ & $\begin{array}{l}0.005 \\
-2890\end{array}$ & $\begin{array}{l}0.0075 \\
-1722\end{array}$ & $\begin{array}{l}0.01 \\
-553\end{array}$ \\
\hline
\end{tabular}

Table 3 Cyclic hardening modulus $Q_{1}\left(\rho_{\max }\right)$ (MPa) as a function of the value of maximal displacement of the yield surface center $\rho_{\max }$ for the heat-resistant alloy Nimonic $80 \mathrm{~A}\left(Q_{2}=0\right)$

\begin{tabular}{lllll}
\hline$T=571^{\circ} \mathrm{C}$ & & & 160.9 & 88.5 \\
\hline$\rho_{\max }$ & 266.6 & 232.7 & 420 & 450 \\
$Q_{1}$ & 384 & 395 & & 63.2 \\
$T=700^{\circ} \mathrm{C}$ & & & 106.6 & 278 \\
\hline$\rho_{\max }$ & 160.5 & 140.9 & 281 & \\
$Q_{1}$ & 303 & 309 & & 45.9 \\
$T=823^{\circ} \mathrm{C}$ & & & 57.86 & 120 \\
\hline$\rho_{\max }$ & 68.7 & 65.8 & 125 & \\
$Q_{1}$ & 230 & 219 & & \\
\hline
\end{tabular}

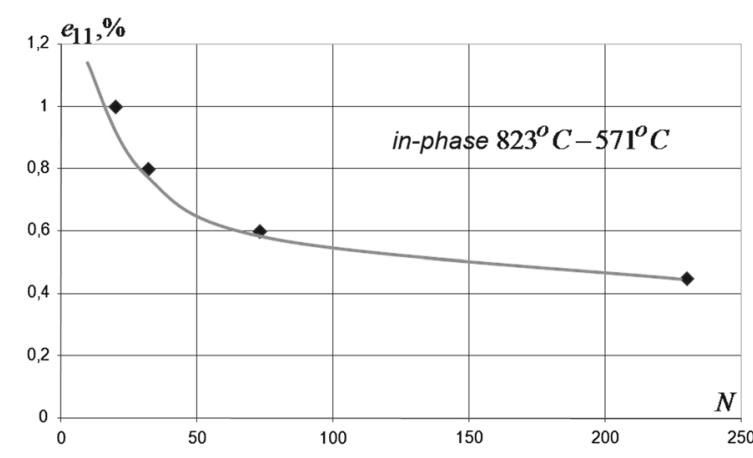

(a)

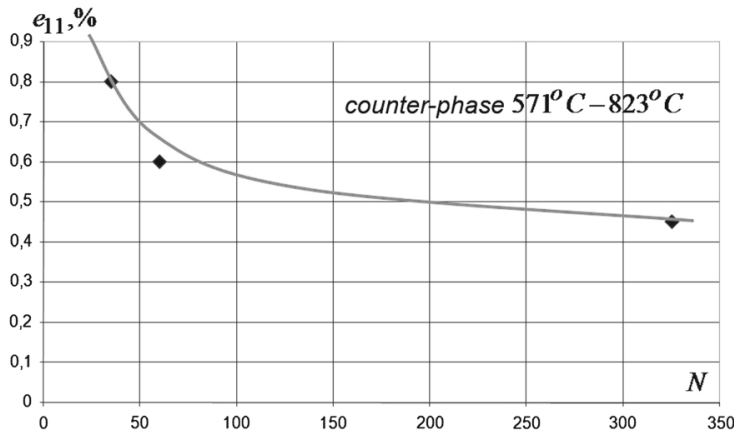

(b)

Fig. 4 Nonisothermal fatigue curves for the in-phase (a) and counter-phase (b) cyclic loading of the heat-resistant alloy Nimonic $80 \mathrm{~A}$

(see Fig. 3), which are used to reconstruct numerically low-cycle fatigue curves for various deformation trajectories with a high accuracy;

- the damaged medium model developed in [12-16] describes accurately enough for engineering purposes the processes of isothermal and nonisothermal deformation and fatigue damage accumulation under combined thermal-mechanical loading and makes it possible to evaluate low-cycle fatigue life of heat-resistant alloys for random deformation trajectories;

- for the isothermal deformation regimes with a strain amplitude of $e_{11}=0.45 \%$, the temperature substantially affects fatigue life of the alloy: fatigue life decreases as the temperature increases (thus, for $T=571{ }^{\circ} \mathrm{C}$ number of cycles to failure $N_{f}=367$, whereas for $T=823^{\circ} \mathrm{C} N_{f}=140$ ). For an increasing strain this trend does not work (see Table 4); 
Table 4 Number of cycles to failure for different types of thermal cyclic loading

\begin{tabular}{|c|c|c|c|c|}
\hline \multirow{2}{*}{\multicolumn{2}{|c|}{ Loading regime }} & \multirow[t]{2}{*}{ Strain amplitude, $e_{11}, \%$} & \multicolumn{2}{|c|}{ Number of cycles to failure, $N_{f}$} \\
\hline & & & Experimental & Numerical \\
\hline \multirow[t]{8}{*}{ Isothermal } & \multirow[t]{4}{*}{$T=571^{\circ} \mathrm{C}$} & 0.45 & 319 & 367 \\
\hline & & 0.6 & 66 & 66 \\
\hline & & 0.8 & 24 & 20 \\
\hline & & 1 & 13 & 10 \\
\hline & \multirow{4}{*}{$T=823^{\circ} \mathrm{C}$} & 0.45 & 128 & 140 \\
\hline & & 0.6 & 65 & 64 \\
\hline & & 0.8 & 51 & 42 \\
\hline & & 1 & 30 & 25 \\
\hline \multirow{4}{*}{\multicolumn{2}{|c|}{ Nonisothermal in-phase }} & 0.45 & 230 & 255 \\
\hline & & 0.6 & 73 & 65 \\
\hline & & 0.8 & 32 & 31 \\
\hline & & 1 & 20 & 17 \\
\hline \multirow{3}{*}{\multicolumn{2}{|c|}{ Nonisothermal counter-phase }} & 0.45 & 325 & 255 \\
\hline & & 0.6 & 60 & 64 \\
\hline & & 0.8 & 35 & 30 \\
\hline
\end{tabular}

- for the nonisothermal deformation regimes in this temperature range $\left(571{ }^{\circ} \mathrm{C}-823^{\circ} \mathrm{C}\right)$ the heat-resistant alloy Nimonic $80 \mathrm{~A}$ is insensitive to the phase pattern of the change in temperature and mechanical deformation (see Table 4).

The next example presents the results of comparing the numerical and experimental data on thermal cyclic deformation of thin-walled tubular specimens of the heat-resistant cobalt alloy Haynes 188 in the temperature interval of $316-760^{\circ} \mathrm{C}$. Isothermal and nonisothermal experiments were conducted with hard loading: the amplitude of axial strain $e_{11}$ was $0.4 \%$, and that of shear strain $e_{12}$ amounted to $0.9 \%$ [26].

In the experiments described in [26], the following parameters varied:

- phase shift angle $\theta$ between axial strain $e_{11}$ and shear strain $e_{12}$ (for isothermal deformation with $\theta=0$ proportional loading; for $\theta=90^{\circ}$, the axial and shear strains vary in counter-phase);

- phase shift angle $\psi$ between the amplitudes of axial strain $e_{11}$ and temperature $\mathrm{T}$ (for nonisothermal loading with $\psi=0$ the axial strain and temperature vary in-phase, for $\psi=180^{\circ}$ in counter-phase).

In the course of complex isothermal and nonisothermal deformation described in [26] eight types of tests were implemented:

- experiment T316MIP: mechanical deformations vary in-phase $(\theta=0)$, temperature $T=316^{\circ} \mathrm{C}$ remains constant (isothermal tests);

- experiment T316MOP: mechanical strains vary in counter-phase $\left(\theta=90^{\circ}\right)$, temperature $T=316^{\circ} \mathrm{C}$ remains constant (isothermal tests);

- experiment T760MIP: mechanical deformations vary in-phase $(\theta=0)$, temperature $T=760^{\circ} \mathrm{C}$ remains constant (isothermal tests);

- experiment T760MOP: mechanical strains vary in counter-phase $\left(\theta=90^{\circ} \mathrm{C}\right)$, temperature $T=760^{\circ} \mathrm{C}$ remains constant (isothermal tests);

- experiment MIPTIP: mechanical strains and temperature vary in-phase $(\theta=0$ and $\psi=0)$-nonisothermal tests;

- experiment MIPTOP: mechanical strains vary in-phase, temperature varies in counter-phase $(\theta=0$ and $\psi=180^{\circ}$ )—nonisothermal tests;

- experiment MOPTIP: mechanical strains vary in counter-phase, temperature varies in-phase $\left(\theta=90^{\circ}\right.$ and $\psi=0$ )—nonisothermal tests;

- experiment MOPTOP: mechanical strains and temperature vary in counter-phase $\left(\theta=90^{\circ}\right.$ and $\psi=$ $180^{\circ}$ )—nonisothermal tests.

Assigning different laws of change of axial deformation $e_{11}$, shear deformation $e_{12}$ and temperature $T$, different deformation trajectories can be realized (see Table 5).

The main physical-mechanical characteristics of the heat-resistant cobalt alloy Haynes 188 [26] and the material parameters of the damaged medium model [12-16] are listed in Tables 6, 7 and 8.

Figure 5 compares the results of numerical modeling and experimental data on the cyclic hysteresis loops for $N=50$-th cycle of nonisothermal loading (experiments MIPTIP, MOPTOP). Here and in what follows, 
Table 5 Laws of change of axial strain $e_{11}$, shear strain $e_{12}$ and temperature $T$ (deformation trajectories)

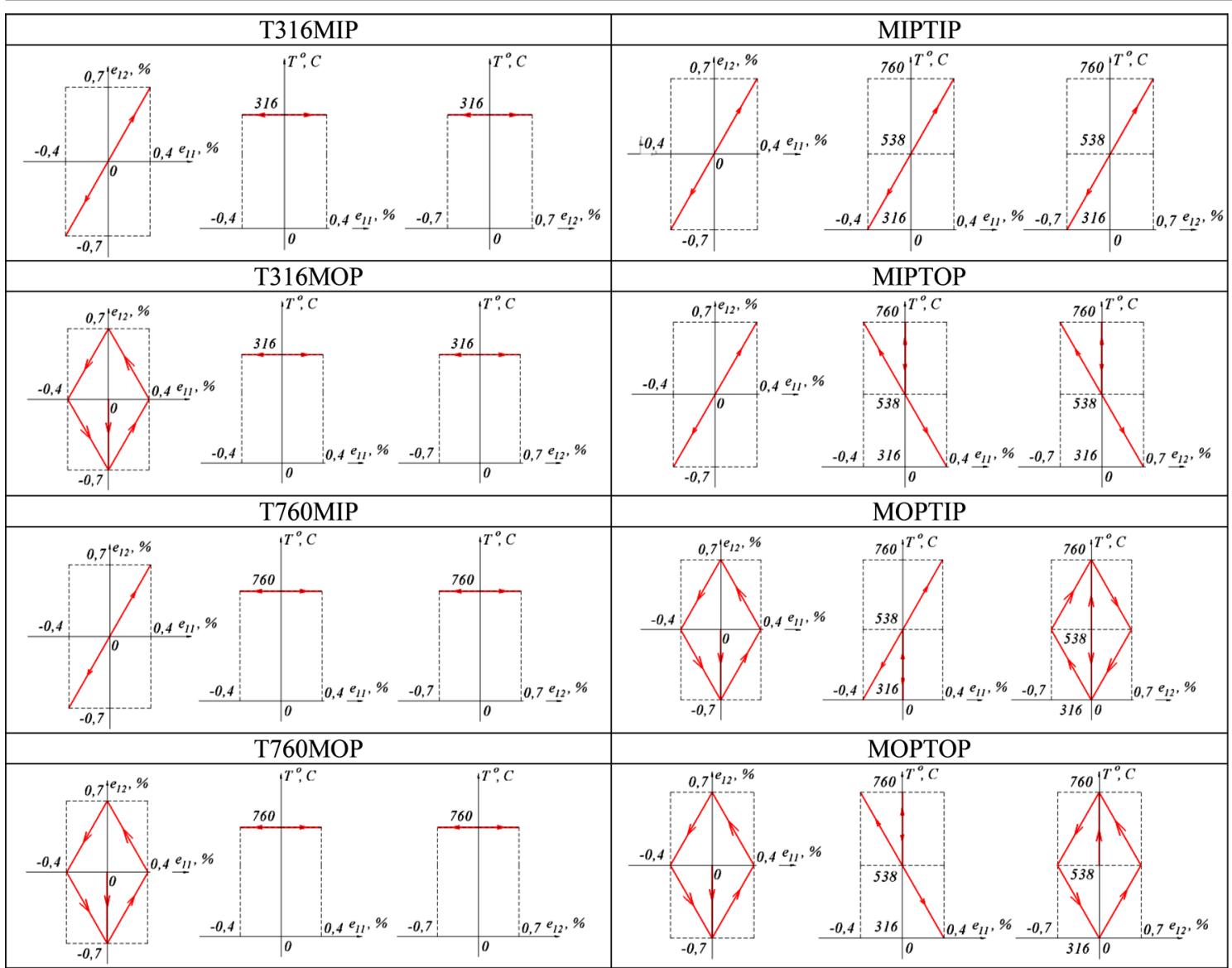

Table 6 The main physical-mechanical characteristics and the material parameters of the damaged medium model for the heat-resistant cobalt alloy Haynes 188

\begin{tabular}{lll}
\hline Characteristics & Temperature $T\left({ }^{\circ} \mathrm{C}\right)$ & 760 \\
\cline { 2 - 3 }$K(\mathrm{MPa})$ & 316 & 138,400 \\
$G(\mathrm{MPa})$ & 167,916 & 63,680 \\
$\alpha(1 / \mathrm{degree})$ & 77,500 & 0.00001531 \\
$C_{p}^{\circ}(\mathrm{MPa})$ & 0.00001319 & 260 \\
$g_{1}(\mathrm{MPa})$ & 295 & 15,770 \\
$g_{2}$ & 18,170 & 182.67 \\
$a$ & 182.74 & 4 \\
$W_{f}\left(\mathrm{MJ} / \mathrm{m}^{3}\right)$ & 4 & 201 \\
$W_{a}\left(\mathrm{MJ} / \mathrm{m}^{3}\right)$ & 1481 & 0 \\
\hline
\end{tabular}

the solid lines indicate the results of numerical modeling using the defining relations of MDM [12-16], and the dotted lines show the corresponding experimental data (the comparison was done for all the test data presented in [26]).

The analysis of the numerical results shows that during isothermal cyclic loading (experiments T316MIP, T316MOP, T760MIP, T760MOP) stabilization of the cyclic thermal-plastic hysteresis loops is observed.

During nonisothermal thermal cyclic loading (experiments MIPTIP, MIPTOP, MOPTIP, MOPTOP) the form of axial $\sigma_{11} \sim e_{11}$ and shear $\sigma_{12} \sim e_{12}$ thermal-plastic hysteresis loops depends on phase shift $\theta$ 
Table 7 Monotone isotropic hardening modulus $q_{1}(\mathrm{MPa})$ as a function of the length of plastic deformation path over monotone parts $\chi_{m}$ for the heat-resistant alloy Haynes $188\left(q_{2}=0\right)$

\begin{tabular}{|c|c|c|c|c|c|c|c|c|c|}
\hline \multicolumn{10}{|c|}{$T=316^{\circ} \mathrm{C}$} \\
\hline $\begin{array}{l}\chi_{m} \\
q_{1}\end{array}$ & $\begin{array}{l}0 \\
-2401 \\
\end{array}$ & $\begin{array}{l}0.0002 \\
-2716 \\
\end{array}$ & $\begin{array}{l}0.0004 \\
-3032 \\
\end{array}$ & $\begin{array}{l}0.0005 \\
-3190 \\
\end{array}$ & $\begin{array}{l}0.001 \\
-3979 \\
\end{array}$ & $\begin{array}{l}0.002 \\
-5296 \\
\end{array}$ & $\begin{array}{l}0.003 \\
-6089 \\
\end{array}$ & $\begin{array}{l}0.005 \\
-6186 \\
\end{array}$ & $\begin{array}{l}0.01 \\
-2133 \\
\end{array}$ \\
\hline \multicolumn{10}{|c|}{$T=760^{\circ} \mathrm{C}$} \\
\hline $\begin{array}{l}\chi_{m} \\
q_{1}\end{array}$ & $\begin{array}{l}0 \\
-2124\end{array}$ & $\begin{array}{l}0.0002 \\
-2129\end{array}$ & $\begin{array}{l}0.0004 \\
-2135\end{array}$ & $\begin{array}{l}0.0005 \\
-2138\end{array}$ & $\begin{array}{l}0.001 \\
-2152\end{array}$ & $\begin{array}{l}0.002 \\
-2470\end{array}$ & $\begin{array}{l}0.003 \\
-3367\end{array}$ & $\begin{array}{l}0.005 \\
-5207\end{array}$ & $\begin{array}{l}0.01 \\
-1471\end{array}$ \\
\hline
\end{tabular}

Table 8 Cyclic hardening modulus $Q_{1}\left(\rho_{\max }\right)(\mathrm{MPa})$ as a function of the maximal displacement value of the yield surface center $\rho_{\max }$ for the heat-resistant alloy Haynes $188\left(Q_{2}=0\right)$

\begin{tabular}{llll}
\hline$T=316^{\circ} \mathrm{C}$ & & & \\
\hline$\rho_{\max }$ & 24.35 & 46.81 & 49.16 \\
$Q_{1}$ & 520 & 520 & 500 \\
\hline$T=760^{\circ} \mathrm{C}$ & & & 60.75 \\
\hline$\rho_{\max }$ & 43.88 & 46.51 & 330 \\
$Q_{1}$ & 350 & 440 & \\
\hline
\end{tabular}
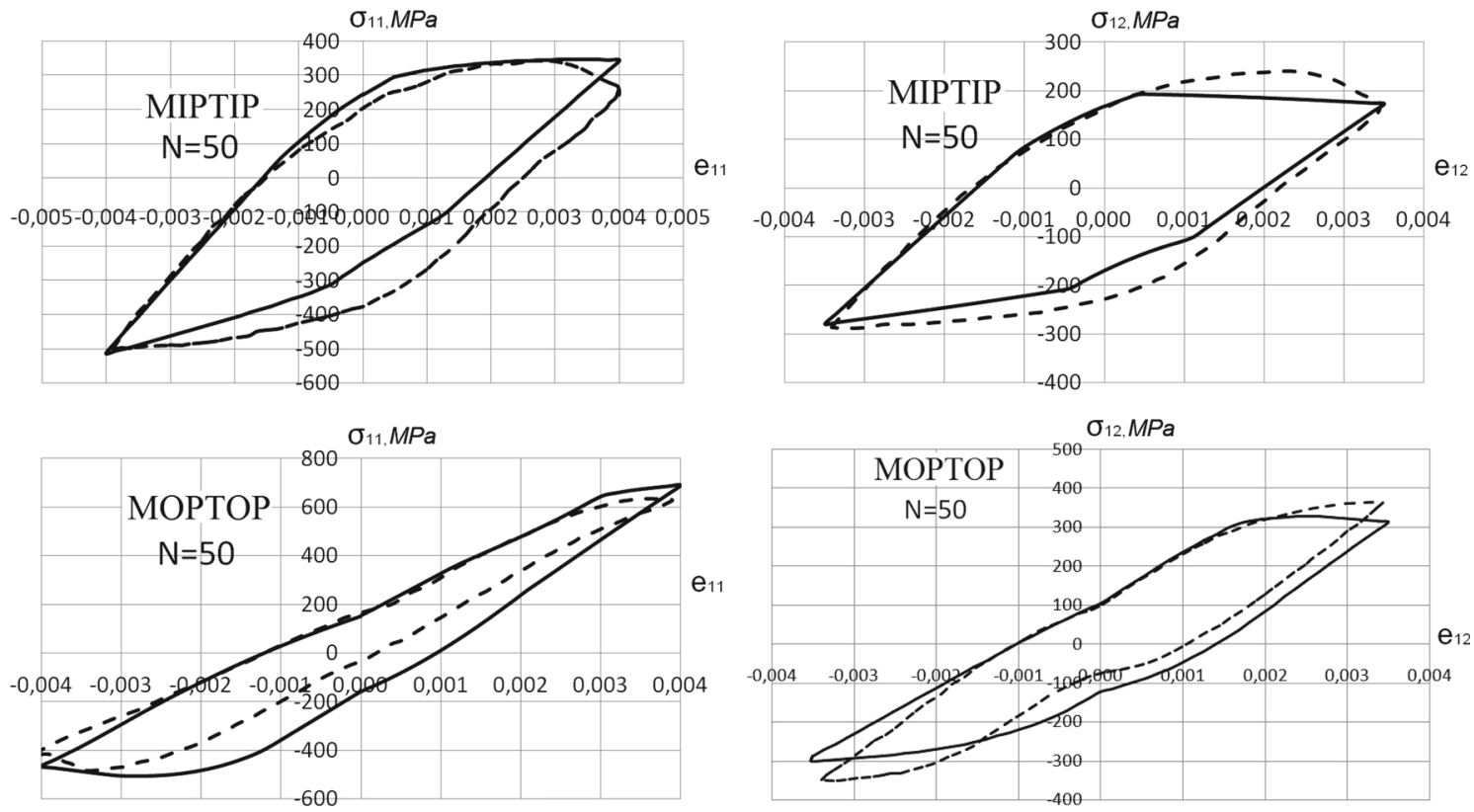

Fig. 5 Results of comparing the experimental and numerical data for cyclic plastic hysteresis loops $(N=50)$ for nonisothermal loading

between the amplitudes of axial strain $e_{11}$ and shear strain $e_{12}$. Thus, in experiment MFTF during the 50-th cycle relaxation of stresses (both normal and tangential) near the maximal temperature of the cycle (see Fig. 5).

The plastic hysteresis loops in experiment MOPTOP qualitatively differ from the in-phase mechanical tests MIPTIP (Fig. 5).

Thus, the position and degree of relaxation of both axial and shear thermal-plastic hysteresis loops are greatly determined by phase shift angle $\psi$ between axial strain $e_{11}$ and temperature $T$.

Figure 6 presents computational and experimental diagrams of the amplitudes of axial $\sigma_{11}$ and shear $\sigma_{12}$ stresses as a function of number of loading cycles. The solid line shows the numerical modeling results; the markers depict the corresponding experimental data. It can be seen that the degree of cyclic hardening for nonisothermal thermal cyclic loading is considerably higher than that observed in the isothermal tests with 


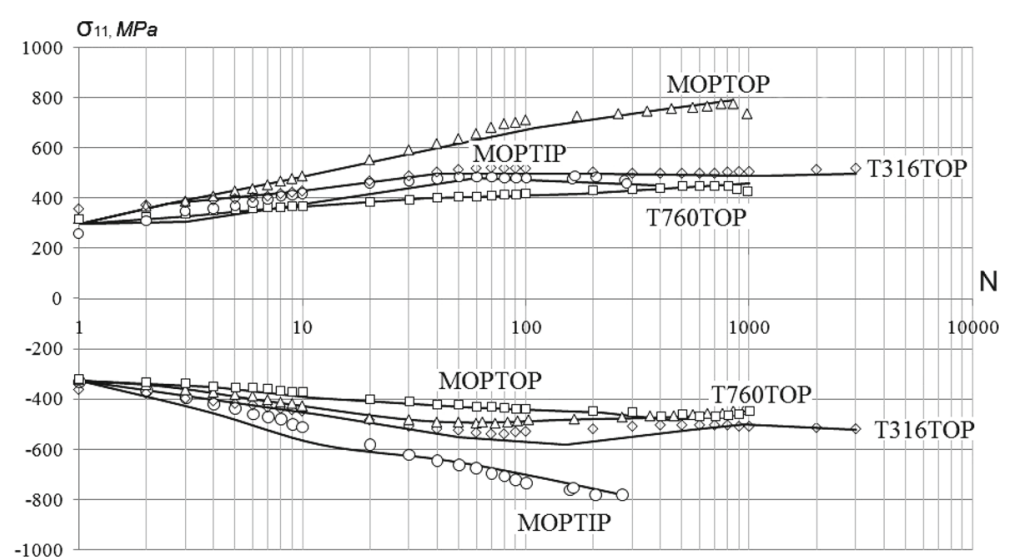

(a)

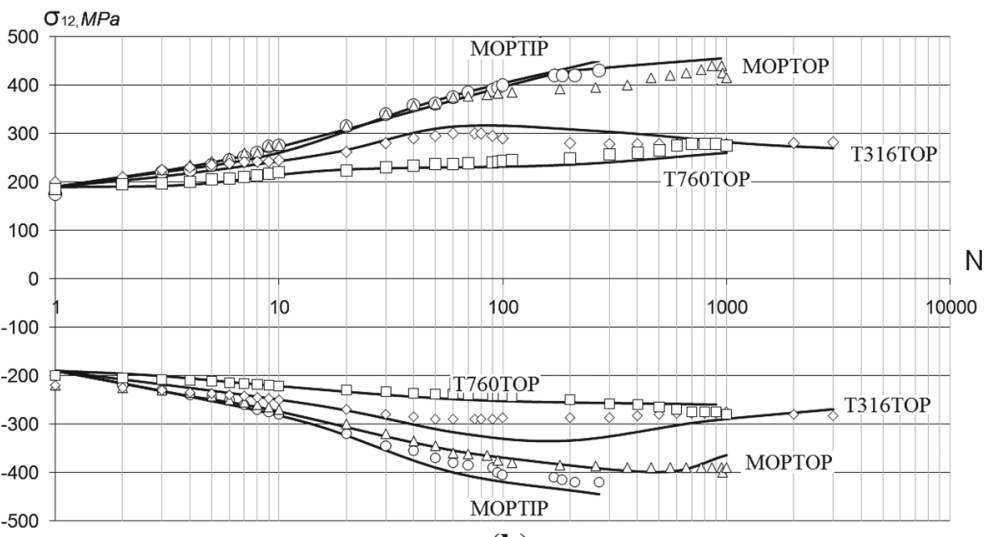

(b)

Fig. 6 Computed and experimental diagrams of the amplitudes of axial $\sigma_{11}$ and shear $\sigma_{12}$ strains

the temperatures of 316 and $760^{\circ} \mathrm{C}$. These observations show that the mechanism of nonisothermal thermal cyclic deformation qualitatively differs from the isothermal one.

The analysis of the obtained results makes it possible to conclude that the model of cyclic thermal-plastic deformation introduced in the paper adequately describes the experimental results and thus can be used in future for analyzing the kinetics of the fatigue damage accumulation process under cyclic thermal-mechanical effects.

To study the effect of deformation trajectory on low-cycle fatigue of heat-resistant alloys, fatigue damage accumulation processes were numerically analyzed for various assigned laws of change of axial strain $e_{11}$, shear strain $e_{12}$ and temperature $T$ (see Table 5). The computational results were compared against the available test data [26]. Figure 7 demonstrates the experimental fatigue curves of the heat-resistant alloy Haynes 188 [27] under uniaxial-tension-compression for temperatures $T=425,650$ and $870{ }^{\circ} \mathrm{C}$, respectively (dotted lines).

The isothermal fatigue curves for temperatures $T=571$ and $760^{\circ} \mathrm{C}$ (solid lines) were obtained using spline-approximation (quadratic spline) of the fatigue curves for temperatures при температурах $T=425,650$ and $870{ }^{\circ} \mathrm{C}$ [27]. To determine material parameters of the evolutionary equation of damage accumulation, point $P$ on the fatigue curves (Fig. 7) was used. The material parameters of the evolutionary equation of damage accumulation (16) for the heat-resistant alloy Haynes 188 are listed in Table 6.

The computational results are presented and compared with the available test data in Table 9.

The comparison of the numerical results and experimental data show that:

- the damaged medium model developed in [12-16] accurately enough for engineering purposes describes the processes of cyclic isothermal and nonisothermal deformation and fatigue damage accumulation under combined thermal-mechanical loading and makes it possible to evaluate low-cycle fatigue life of heatresistant alloys for arbitrary deformation trajectories (when comparing experimental and test data it should 


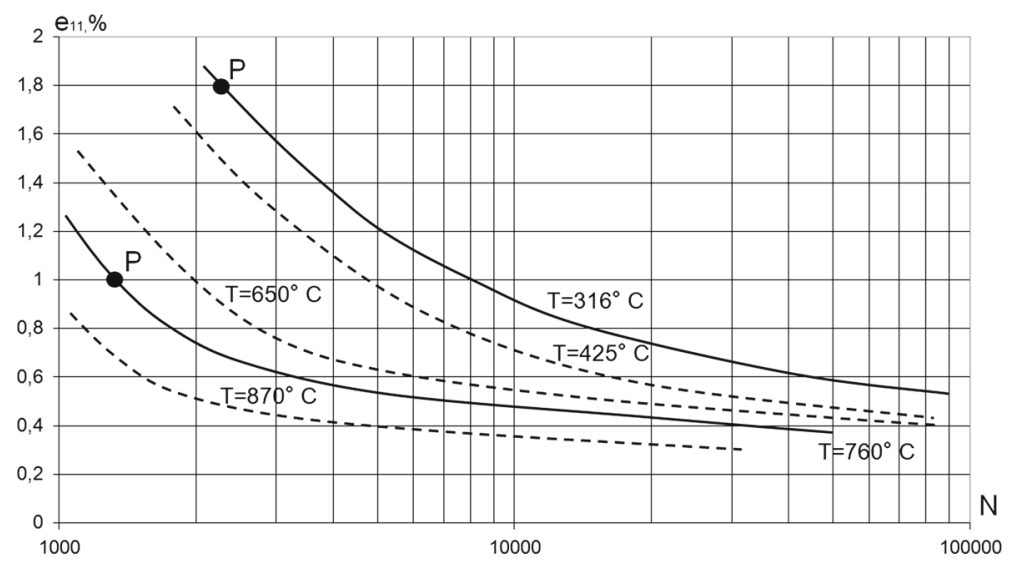

Fig. 7 Fatigue curves of heat-resistant alloy Haynes 188 under isothermal loading

Table 9 Number of cycles to loading for different regimes of thermal cyclic loading

\begin{tabular}{lcr}
\hline Loading regime & Number of loading cycles, $N$ & Numerical \\
\cline { 3 - 3 } & Experiment & 10,512 \\
\hline Isothermal & & 2860 \\
T316MIP & 9448 & 1205 \\
T316MOP & 3410 & 785 \\
T760MIP & 910 & 143 \\
T760MOP & 1089 & 830 \\
Isothermal & 282 & 341 \\
MIPTIP & 569 & 1307 \\
MIPTOP & 270 & \\
MOPTIP & 1004 & \\
MOPTOP & & \\
\hline
\end{tabular}

be kept in mind that the information in [26] is presented without accounting for the scatter of experimental data within each individual test);

- during isothermal cyclic thermal-plastic deformation $T=316^{\circ} \mathrm{C}$, nonproportional mechanical deformation (test T316MOP) leads to an almost threefold decrease in fatigue life as compared with the proportional one (test T316MIP). With the increasing temperature $T=760^{\circ} \mathrm{C}$ this dependence does not work;

- nonisothermal cyclic thermal-plastic deformation leads to an increased fatigue life as compared with the isothermal one;

- the lowest fatigue life is observed when the maximal value of the temperature cycle is achieved in a halfcycle of tension for mechanical strains $e_{11}$ and $e_{12}$, whereas the minimal value of the temperature cycle is achieved in a half-cycle of compression (test MIPTIP), which agrees with the experimental data of [26]. The highest fatigue life (test MOPTOP) is observed when mechanical strains and temperature vary in counter-phase $\left(\theta=90^{\circ}\right.$ and $\left.\psi=180^{\circ}\right)$.

\section{Conclusions}

In the framework of mechanics of damaged media, a mathematical model has been developed, which describes processes of thermal-plastic deformation and fatigue damage accumulation in polycrystalline structural alloys under arbitrary complex regimes of low-cycle loading

The reliability of the modes has been assessed by comparing the computational results with the test data on thermal cyclic fatigue life of heat-resistant alloys (Nimonic 80A, Haynes 188) under isothermal and nonisothermal regimes of combined thermal-mechanical loading, which corroborated the adequacy of modeling and determining of the material parameters of the defining relations of MDM.

Some interesting analyses of damage and fracture can be carried with very performing results also in case of microstructured architectured materials. These materials exhibit different architectures and geometries. A large class of them can be considered as constituted by fibers, which may be modeled as Euler-Bernoulli beams 
[28-32]. In particular, one can develop continuum models for the description of fiber reinforced materials, as [33], or array of fibers planarly aligned, also known as pantographic structures [34-44]. For 2D lattices with uniform triangular microarchitecture (being possibly the simplest possible 2D configuration and providing an isotropic material in the classical sense), the material properties related to bending has been determined in [45]. For a more complicated 2D triangular lattice, higher-order material parameters associated with the third gradient elasticity, have been determined by tension, shear and vibration tests in [46]. Before studying damage and fracture in generalized material, it will be useful to perform some dynamic analyses as the one presented in $[47,48]$. In particular, first experimental results about dynamic behavior in pantographic metamaterials has been discussed in [49].

Analysis of damage and fracture can be also performed in the field of generalized materials, suitably described by higher gradient models. Some properties of higher gradient models can be involved in such analyses. In particular, it is possible to refer to [50-52] for detailed descriptions of these models and their principal features when the considered material is composed by inextensible fibers. A variational approach for a nonlinear one-dimensional damage-elasto-plastic second-gradient continuum model is reported in [53].

Strain gradient modeling has proven to be useful also in regularizing mesh-dependent problems in continuum damage and fracture mechanics, where localization of deformations is frequently observed. Implementation of such regularization in second-gradient materials would be straightforward, also in view of parameters identification carried out in [54-57]. In [58] experimental results relative to displacement-controlled large deformation shear loading tests of pantographic structures are reported. A generalized plate model is validated by means of these experimental results. In particular, five differently sized samples are analyzed up to first rupture. Results show that the deformation behavior is strongly nonlinear, and the structures are capable of undergoing large elastic deformations without reaching complete failure.

The thermo-mechanical approaches are, in general, very useful. Apart the applications we have studied in this paper, these methods can be suitably applied also in case of beams. An independent and novel approach to understand the large deformation of beams consists in regarding a beam as the boundary curve of a twodimensional manifold in a three-dimensional space. In doing so, not only is the curve endowed with its own energy similar to that in the context of lower-dimensional energetics [59] but also in a geometrically nonlinear framework [60] and in accordance with higher gradient elasticity accounting for boundary energetics elaborated in [61]. The advantage of this approach, particularly from a computational viewpoint, is that the bulk material acts to regularize the behavior of the beam especially important to analyze the instabilities associated with thin beams similar to the instabilities of thin films on an elastic foundation [62]. Obviously, in the limiting case of the vanishing bulk one would recover exactly the beam theory.

In the field of polycrystalline structural alloys, a further application of the methods developed in this paper could be could be implemented in the case of the Potts model, whose 1D case is exactly solvable [63,64].

The above-discussed methods, especially for generalized materials, can be validated by numerical simulations. One of the currently most adopted techniques in finite elements coding is based on isogeometric analyses [65-70].

Acknowledgements This work was supported by a grant from the Government of the Russian Federation (Contract No. 14.Y26.31.0031).

Open Access This article is distributed under the terms of the Creative Commons Attribution 4.0 International License (http:// creativecommons.org/licenses/by/4.0/), which permits unrestricted use, distribution, and reproduction in any medium, provided you give appropriate credit to the original author(s) and the source, provide a link to the Creative Commons license, and indicate if changes were made.

\section{References}

1. Halford, G.R.: Low cycle thermal fatigue. Mechanics and Mathematical Methods. In: Thermal Stress II. Chapter 6. pp. 329-428. Elsevier (1987)

2. Serencen, S.V. (ed.): Issledovanie malotsiklovoy prochnosti pri vysokih temperaturah. (in Russ.). Nauka, Moscow (1975)

3. Collins, J.A.: Damage of Materials in Structures. Analysis, Prediction, Prevention. Wiley, New York (1981)

4. Eorum: Otsenka sovremennoy metodologii proektirovaniya vysokotemperaturnyh elementov konstruktsiy na osnove eksperimentov po ih razrusheniyu (in Russ.) Teoreticheskiye osnovy inzhenernyh raschetov. 11 pp. 104-118 (1988)

5. Bernard-Connolly, M., Biron, A., Bue-Quic, T.: Low-cycle fatigue behaviour and cumulative dormage effect of SA-51670 steel at room and high temperature. In: 4th International Conference Pressure Vessel Technology, vol. 1, pp. 297-302. Institution of Mechanical Engineers, London (1980)

6. Gusenkov, A.P., Eazantsev, A.G.: Prochnost pri malotsiklovom i dlitelnom tsiklicheskom nagruzhesnii i nagreve (in Russ.). Mashinovedenie 3, 59-65 (1979) 
7. Sentoglou: Vliyanie ogranicheniy na termomehanicheskuyu ustalost (in Russ.) Teoreticheskie osnovy inzhenernyh raschetov. 3, 74-83 (1985)

8. Bodner, S.R., Lindholm, U.S.: An incremental criterion for time-dependent failure of materials. J. Eng. Mater. Technol. ASME 98(2), 140-145 (1976)

9. Lemaitre, J., et al.: A continuous damage mechanics model for ductile fracture. J. Eng. Mater. Technol. ASME 107(1), 83-89 (1985)

10. Murakami, S., Imaizumi, T.: Mechanical description of creep damage and its experimental verification. J. Méc. Théor. Appl. 1, 743-761 (1982)

11. Chaboche, J.L.: Continuous damage mechanics a tool to describe phenomena before crack initiation. Eng. Des. 64, 233-247 (1981)

12. Volkov, I.A., Eorotkikh, Y.G.: Uravneniya sostoyaniya vyazkouprugoplasticheskih sred s povrezhdeniyami (in Russ.). Fizmatlit, Moscow (2008)

13. Volkov, I.A., Igumnov, L.A.: Vvedenie v kontinualnuyu mehaniku povrezhdennoy sredy (in Russ.). Fizmatlit, Moscow (2017)

14. Volkov, I.A., Eorotkikh, Y.G., Tarasov, I.S.: Modelirovanie slozhnogo plasticheskogo deformirovaniya i razrusheniya metallov pri mnogoosnom neproportsionalnom nagruzhenii (in Russ.). PMTF 50(5), 193-205 (2009)

15. Volkov, I.A., Eorotkikh, Yu.G., Tarasov, I.S.: Chislennoe modelirovanie nakopleniya povrezhdeniy pri slozhnom plasticheskom deformirovanii (in Russ.) Vychisl. meh. splosh. sred. 2(1), 5-18 (2009)

16. Volkov, I.A., Shishulin, D.N., Eazakov, D.A., Pichkov, S.N.: Modelirovanie osnovnyh zakonomernostey protsessa deformirovaniya i nakopleniya povrezhdeniy v konstruktsionnyh materialah na baze kontseptsii mehaniki povrezhdennoi sredy (in Russ.). Problemy prochnosti i plastichnosti 74, 16-27 (2012)

17. Barchiesi, E., Spagnuolo, M., Placidi, L.: Mechanical metamaterials: a state of the art. Math. Mech. Solids (2018). https:// doi.org/10.1177/1081286517735695

18. di Cosmo, F., Laudato, M., Spagnuolo, M.: Acoustic metamaterials based on local resonances: homogenization, optimization and applications. In: Generalized Models and Non-classical Approaches in Complex Materials 1 (pp. 247-274). Springer, Cham (2018)

19. Cuomo, M.: Continuum damage model for strain gradient materials with applications to 1D examples. Contin. Mech. Thermodyn. 1-19 (2018). https://doi.org/10.1007/s00161-018-0698-7

20. Cuomo, M.: Continuum model of microstructure induced softening for strain gradient materials. Math. Mech. Solids (2018). https://doi.org/10.1177/1081286518755845

21. Spagnuolo, M., Barcz, K., Pfaff, A., Dell'Isola, F., Franciosi, P.: Qualitative pivot damage analysis in aluminum printed pantographic sheets: numerics and experiments. Mech. Res. Commun. 83, 47-52 (2017)

22. Battista, A., Rosa, L., dell'Erba, R., Greco, L.: Numerical investigation of a particle system compared with first and second gradient continua: deformation and fracture phenomena. Math. Mech. Solids 22(11), 2120-2134 (2017)

23. Mitenkov, F.M., Volkov, I.A., Igumnov, L.A., Eorotkikh, Y.G., Panov, V.A.: Prikladnaya teoriya plastichnosti (in Russ.). Fizmatlit, Moscow (2015)

24. Volkov, I.A., Igumnov, L.A., Shishulin, D.N., Tarasov, I.S., Markova, M.T.: Modelirovanie ustalostnoy dolgovechnosti polikristallicheskih konstruktsionnyh splavov pri blochnom nesimmetrichnom malotsilkovom nagruzhenii (in Russ.). Problemy prochnosti i plastichnosti 80(1), 15-30 (2018)

25. Liang, J., Pellox, R.M., Xie, X.: Thermomechanical fatique behavior of a nickel base superalloy. Chin. J. Met. Sci. Technol. 5, 1-7 (1989)

26. Kalluri, S., Bonacuse, P.J.: An axial-torsional termomechanical fatique testing technique. Preparade for the Symposium on multiaxial fatique and deformation testing techniques. Denver, Colorado, 25 p (1995)

27. HAYNES ${ }^{1} 188$ ALLOY. STANDART PRODUCTS by Brand or Alloy Designation H-3001B/Global Headquarters, Kokomo, Indiana, USA

28. Andreaus, U., Spagnuolo, M., Lekszycki, T., Eugster, S.R.: A Ritz approach for the static analysis of planar pantographic structures modeled with nonlinear Euler-Bernoulli beams. Contin. Mech. Thermodyn. 30, 1103-1123 (2018)

29. Spagnuolo, M., Andreaus, U.: A targeted review on large deformations of planar elastic beams: extensibility, distributed loads, buckling and post-buckling. Math. Mech. Solids (2018). https://doi.org/10.1177/1081286517737000

30. Eugster, S.R., Glocker, C.: Constraints in structural and rigid body mechanics: a frictional contact problem. Ann. Solid Struct. Mech. 5(1-2), 1-13 (2013)

31. Eugster, S., Glocker, C.: Determination of the transverse shear stress in an Euler-Bernoulli beam using non-admissible virtual displacements. PAMM 14(1), 187-188 (2014)

32. Eugster, S.R.: An intrinsic geometric formulation of the equilibrium equations in continuum mechanics. PAMM 15(1), 289-290 (2015)

33. Franciosi, P., Spagnuolo, M., Salman, O.U.: Mean Green operators of deformable fiber networks embedded in a compliant matrix and property estimates. Contin. Mech. Thermodyn. 31, 101-132 (2019)

34. Boutin, C., Giorgio, I., Placidi, L.: Linear pantographic sheets: asymptotic micro-macro models identification. Math. Mech. Complex Syst. 5(2), 127-162 (2017)

35. Misra, A., Lekszycki, T., Giorgio, I., Ganzosch, G., Müller, W.H., dell'Isola, F.: Pantographic metamaterials show atypical Poynting effect reversal. Mech. Res. Commun. 89, 6-10 (2018)

36. Turco, E., Golaszewski, M., Giorgio, I., D’Annibale, F.: Pantographic lattices with non-orthogonal fibres: experiments and their numerical simulations. Compos. Part B Eng. 118, 1-14 (2017)

37. Giorgio, I., Rizzi, N.L., Turco, E.: Continuum modelling of pantographic sheets for out-of-plane bifurcation and vibrational analysis. Proc. R. Soc. A 473(2207), 20170636 (2017)

38. Placidi, L., Barchiesi, E., Turco, E., Rizzi, N.L.: A review on 2D models for the description of pantographic fabrics. Z. Angew. Math. Phys. 67(5), 121 (2016)

39. Barchiesi, E., Placidi, L.: A review on models for the 3D statics and 2D dynamics of pantographic fabrics. In: Wave Dynamics and Composite Mechanics for Microstructured Materials and Metamaterials (pp. 239-258). Springer, Singapore (2017) 
40. Placidi, L., Andreaus, U., Giorgio, I.: Identification of two-dimensional pantographic structure via a linear D4 orthotropic second gradient elastic model. J. Eng. Math. 103(1), 1-21 (2017)

41. Placidi, L., Barchiesi, E., Battista, A.: An inverse method to get further analytical solutions for a class of metamaterials aimed to validate numerical integrations. In: Mathematical Modelling in Solid Mechanics (pp. 193-210). Springer, Singapore (2017)

42. Turco, E., Golaszewski, M., Cazzani, A., Rizzi, N.L.: Large deformations induced in planar pantographic sheets by loads applied on fibers: experimental validation of a discrete Lagrangian model. Mech. Res. Commun. 76, 51-56 (2016)

43. Golaszewski, M., Grygoruk, R., Giorgio, I., Laudato, M., Di Cosmo, F.: Metamaterials with relative displacements in their microstructure: technological challenges in 3D printing, experiments and numerical predictions. Contin. Mech. Thermodyn. 1-20 (2018). https://doi.org/10.1007/s00161-018-0692-0

44. Lekszycki, T., Di Cosmo, F., Laudato, M., Vardar, O.: Application of energy measures in detection of local deviations in mechanical properties of structural elements. Contin. Mech. Thermodyn. 31(2), 413-425 (2019)

45. Khakalo, S., Balobanov, V., Niiranen, J.: Modelling size-dependent bending, buckling and vibrations of 2D triangular lattices by strain gradient elasticity models: applications to sandwich beams and auxetics. Int. J. Eng. Sci. 127, 33-52 (2018)

46. Khakalo, S., Niiranen, J.: Form II of Mindlin's second strain gradient theory of elasticity with a simplification: for materials and structures from nano- to macro-scales. Eur. J. Mech. A/Solids 71, 292-319 (2018)

47. Abbas, I.A., Abdalla, A.E.N.N., Alzahrani, F.S., Spagnuolo, M.: Wave propagation in a generalized thermoelastic plate using eigenvalue approach. J. Therm. Stress. 39(11), 1367-1377 (2016)

48. Abd-alla, A.E.N.N., Alshaikh, F., Del Vescovo, D., Spagnuolo, M.: Plane waves and eigenfrequency study in a transversely isotropic magneto-thermoelastic medium under the effect of a constant angular velocity. J. Therm. Stress. 40(9), 1079-1092 (2017)

49. Laudato, M., Manzari, L., Barchiesi, E., Di Cosmo, F., Göransson, P.: First experimental observation of the dynamical behavior of a pantographic metamaterial. Mech. Res. Commun. 94, 125-127 (2018)

50. Mohammed, E.S., Placidi, L.: Discrete and continuous aspects of some metamaterial elastic structures with band gaps. Arch. Appl. Mech. 18, 1725-1742 (2018). https://doi.org/10.1007/s00419-018-1399-1. ISSN: 0939-1533

51. Placidi, L., Dhaba, A.R.E.: Semi-inverse method à la Saint-Venant for two-dimensional linear isotropic homogeneous second gradient elasticity. Math. Mech. Solids 22, 919-937 (2017). https://doi.org/10.1177/1081286515616043. ISSN: 1081-2865

52. Placidi, L., Greco, L., Bucci, S., Turco, E., Rizzi, N.L.: A second gradient formulation for a 2D fabric sheet with inextensible fibres. Z. Angew. Math. Phys. 67, 114 (2016). https://doi.org/10.1007/s00033-016-0701-8. ISSN: 0044-2275

53. Placidi, L.: A variational approach for a nonlinear one-dimensional damage-elasto-plastic second-gradient continuum model. Contin. Mech. Thermodyn. 28, 119-137 (2016). https://doi.org/10.1007/s00161-014-0405-2. ISSN: 0935-1175

54. Placidi, L., Barchiesi, E.: Energy approach to brittle fracture in strain-gradient modelling. Proc. R. Soc. A 474(2210), 20170878 (2018)

55. Placidi, L., Misra, A., Barchiesi, E.: Two-dimensional strain gradient damage modeling: a variational approach. Z. Angew. Math. Phys. 69(3), 56 (2018)

56. Placidi, L., Barchiesi, E., Misra, A.: A strain gradient variational approach to damage: a comparison with damage gradient models and numerical results. Math. Mech. Complex Syst. 6(2), 77-100 (2018)

57. Placidi, L., Misra, A., Barchiesi, E.: Simulation results for damage with evolving microstructure and growing strain gradient moduli. Contin. Mech. Thermodyn. 1-21 (2018). https://doi.org/10.1007/s00161-018-0693-z

58. Barchiesi, E., Ganzosch, G., Liebold, C., Placidi, L., Grygoruk, R., Müller, W.H.: Out-of-plane buckling of pantographic fabrics in displacement-controlled shear tests: experimental results and model validation. Contin. Mech. Thermodyn. 1-13 (2018). https://doi.org/10.1007/s00161-018-0626-x

59. Javili, A., Mcbride, A., Steinmann, P.: Thermomechanics of solids with lower-dimensional energetics: On the importance of surface, interface, and curve structures at the nanoscale. A unifying review. Appl. Mech. Rev. 65, 010802 (2013)

60. Javili, A., McBride, A., Steinmann, P., Reddy, B.D.: A unified computational framework for bulk and surface elasticity theory: a curvilinear-coordinate-based finite element methodology. Comput. Mech. 54, 745-762 (2014)

61. Javili, A., dell'Isola, F., Steinmann, P.: Geometrically nonlinear higher-gradient elasticity with energetic boundaries. J. Mech. Phys. Solids 61, 2381-2401 (2013)

62. Javili, A., Dortdivanlioglu, B., Kuhl, E., Linder, C.: Computational aspects of growth-induced instabilities through eigenvalue analysis. Comput. Mech. 56, 405-420 (2015)

63. De Masi, A., Merola, I., Presutti, E., Vignaud, Y.: Potts models in the continuum. Uniqueness and exponential decay in the restricted ensembles. J. Stat. Phys. 133(2), 281-345 (2008)

64. De Masi, A., Merola, I., Presutti, E., Vignaud, Y.: Coexistence of ordered and disordered phases in Potts models in the continuum. J. Stat. Phys. 134(2), 243-306 (2009)

65. Niiranen, J., Kiendl, J., Niemi, A.H., Reali, A.: Isogeometric analysis for sixth-order boundary value problems of gradientelastic Kirchhoff plates. Comput. Methods Appl. Mech. Eng. 316, 328-348 (2017)

66. Khakalo, S., Niiranen, J.: Isogeometric analysis of higher-order gradient elasticity by user elements of a commercial finite element software. Comput. Aided Des. 82, 154-169 (2017)

67. Greco, L., Cuomo, M., Contrafatto, L., Gazzo, S.: An efficient blended mixed B-spline formulation for removing membrane locking in plane curved Kirchhoff rods. Comput. Methods Appl. Mech. Eng. 324, 476-511 (2017)

68. Cuomo, M., Greco, L.: An implicit strong G1-conforming formulation for the analysis of the Kirchhoff plate model. Contin. Mech. Thermodyn. 1-25 (2018). https://doi.org/10.1007/s00161-018-0701-3

69. Cazzani, A., Malagù, M., Turco, E.: Isogeometric analysis of plane-curved beams. Math. Mech. Solids 21(5), 562-577 (2016)

70. Cazzani, A., Atluri, S.N.: Four-noded mixed finite elements, using unsymmetric stresses, for linear analysis of membranes. Comput. Mech. 11(4), 229-251 (1993)

Publisher's Note Springer Nature remains neutral with regard to jurisdictional claims in published maps and institutional affiliations. 